\title{
Personalized Web Explore with Disguised User Contour Structure
}

\section{A. Laxmiprasanna, Srivalli}

\begin{abstract}
- site structures square measure altered to boost the user navigations. net personification methodology reconstructs the page relates with relevance the traversal path and contour of a particular user. Users knowledge square measure collected and analyzed to fetch the intention behind the issued question. User customizable Privacy protective Explore (UPS) is employed to generalize contours by queries with user privacy necessities. Greedy discriminating power algorithmic program (GreedyDP) is employed to maximise the discriminating power of the user contours. Greedy knowledge Loss (GreedyIL) is employed to attenuate the info loss in user contours. GreedyIL algorithmic program achieves high potency than the GreedyDP algorithmic program. The Personification net Explore (PWS) theme is increased to manage topic relationship based mostly knowledgeable attacks. The User customizable Privacy-preserving Explore (UPS) model is increased to resist question session based mostly attacks. question generalization is performed with question priority values. Anonymisation and topic taxonomy models square measure wont to improve the personification method.
\end{abstract}

Index Terms: User customizable Privacy-preserving Explore,Privacy protection, personification web explore, GreedyData Loss and Query generalization

\section{INTRODUCTION}

Because the quantity of knowledge on the online unceasingly grows, it's become progressively troublesome for net explore engines to seek out knowledge that satisfies users' individual desires. Personification explore may be a promising thanks to improve explore quality by customizing explore results for individuals with totally different knowledge goals. many recent reexplore efforts have centered on this space. Most of them may be classified into 2 general approaches: Re-ranking question results obtained by explore engines domestically mistreatment personal knowledge; or causation personal data and queries along to the explore engine. an honest personification algorithmic program depends on made user contours and net corpus.

However, because the net corpus is on the server, re-ranking on the consumer aspect is information measure intensive as a result of it needs an oversized range of explore results transmitted to the consumer before re-ranking. or else, if the quantity of knowledge transmitted is restricted through filtering on the server aspect, it pins high hope on the existence of desired knowledge among filtered results, that isn't continuously the case.

Revised Manuscript Received on 14 August, 2019.

A.Laxmiprasanna, Assistant Professor, Department of Computer Science and Engineering, Malla Reddy Engineering College for Women, Maisammaguda, Hyderabad. laxmiprasanna1992@gmail.com

Srivalli, Assistant Professor, Department of Computer Science and Engineering, Malla Reddy Engineering College for Women, Maisammaguda, Hyderabad sri20mrecw@gmail.com
Therefore, most of personification explore services on-line like Google Personification Explore and Yahoo! My net adopts the second approach to tailor results on the server by analyzing collected personal knowledge, e.g. personal interests, and explore histories. Personal knowledge, i.e. browsing history, emails, etc., square measure largely unstructured, that it's onerous to live privacy.

In addition, it's additionally troublesome to include unstructured knowledge with explore engines while not account. So, for the aim of each net personification and privacy preservation, it's necessary for AN algorithmic program to gather, summarize, and organize a user's personal knowledge into a structured user contour. Meanwhile, the notion of privacy is very subjective and depends on the people concerned.

Things thought-about to be non-public by one person may be one thing that others would like to share. during this regard, the user ought to have management over that components of the user contour is shared with the server. Web Mining, that focuses on mechanically discovering knowledge and data through the analysis of net contents, net structure and net usages.

Since the online is large, heterogeneous and dynamic, machine-driven net knowledge and data discovery concerns novel technologies and tools, which can cash in of the progressive technologies from numerous areas, as well as machine learning, data processing, knowledge retrieval, information and nature language process. the online itself and also the explore engine indices contain knowledge regarding the documents. Documents have differing kinds of relationships among themselves.

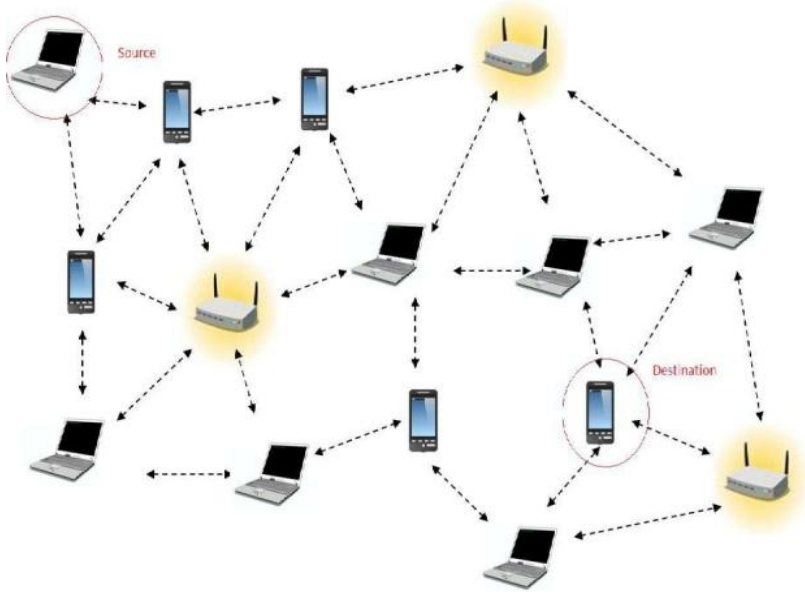

Hyperlinks add depth to documents, providing the multi-dimensionality, that characterizes the online. Documents have AN address, a URL, that represents a logical location on a server, which can offer knowledge regarding the connection of this document to different on the server. 


\section{Personalized Web Explore with Disguised User Contour Structure}

Also, there's a relationship to different documents on the online unknown to the document, the explore engine index might discover such relationships.

\section{RELATED WORK}

Web mining may be a immense, knowledge domain and extremely dynamic scientific space, connection from many reexplore communities like information, knowledge retrieval, and computer science particularly from machine learning and language process. connected add knowledge retrieval, a lot of reexplore is concentrated on personification explore. connectedness feedback and question refinement harnesses a short model of a user's interests, and knowledge a couple of user's intent is collected at question time.

Personal knowledge has additionally been utilized in the context of net explore. There square measure still approaches, as well as several commercially offered data-filtering systems, that need users expressly specify their interests. However, as noted, users square measure generally unwilling to pay the additional effort on specifying their intentions.

Even if they're intended, they're not continuously undefeated in doing thus. A majority of labor focuses on implicitly building user contours to infer a user's intention. a good vary of implicit user activities are projected as sources of increased explore knowledge. This includes a user's explore history, browsing history, click-through knowledge, net community, and made consumer aspect knowledge within the style of desktop indices.

Our approach is receptive all types of various knowledge sources for building user contours, provided the sources are often extracted into text. In our experiments knowledge sources like i.e. histories, emails and up to date personal documents were tested. User contours are often pictured by a weighted term vector, weighted conception class-conscious structures like ODP3, or different implicit user interest hierarchy.

For the needs of by selection exposing users' interests to explore engines, the user contour may be a term $\{$ based|based mostly|primarily based mostly\} data structure that's associated with frequent term based agglomeration algorithms. The distinction here is that the data structure is implicitly made in an exceedingly top-down fashion. and also the focus is that the relationships among terms, not agglomeration the terms into teams. Privacy issues square measure natural and vital particularly on the net.

Some previous studies on non-public knowledge Retrieval $\& \# 40 ;$ PIR\&\#41; , focuses on the matter of permitting the user to retrieve knowledge whereas keeping the question non-public. Instead, this study targets protective privacy of the user contour, whereas still profiting from selective access to general knowledge that the user agrees to unharness. To our data, this drawback has not been studied within the context of personification explore. One potential reason for this is often that non-public knowledge, i.e.

browsing history and emails, is usually unstructured knowledge, that privacy is troublesome to live and quantify. Some works on privacy problems within the data processing community concentrate on protective individual knowledge entries whereas permitting knowledge account. a preferred approach of measure privacy in data processing is by examining the distinction in previous and posterior data of a particular price.

This can be formalized because the chance or Shannon's knowledge theory. in our own way to live privacy is that the notion of k-anonymity that advocates that in person distinctive attributes be generalized such every person is indistinguishable from a minimum of $\mathrm{k}-1$ different persons. In this study the notion of privacy doesn't compare knowledge from totally different users, however rather the info collected over time for one user. additionally, this study addresses unstructured knowledge.

\section{DRABACK DEFINITION}

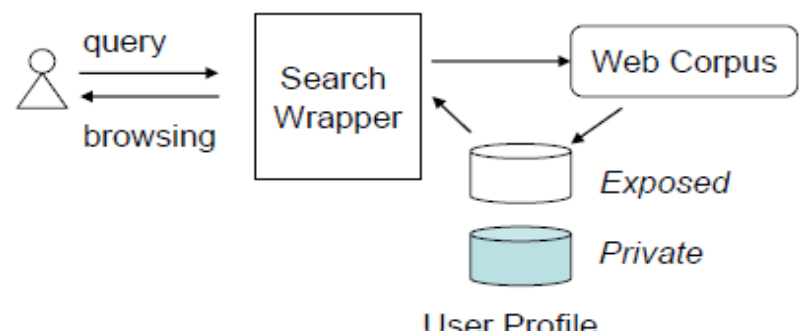

Figure 1: System Overview

Personal knowledge, i.e. personal documents, browsing history and emails may well be useful to spot a user's implicit intents.

However, users have issues regarding however their personal knowledge is employed. Privacy, as against security or confidentiality, extremely depends on the person concerned and the way that person might get pleasure from sharing personal knowledge. The question here is whether or not an answer are often found wherever users themselves square measure able to set their own privacy levels for user contours to boost the explore quality.

/ Figure one: System summary Figure 1 provides an outline of the entire system. AN algorithmic program is provided for the user to mechanically build a class-conscious user contour that represents the user's implicit personal interests. General interests square measure placed on a better level; specific interests square measure placed on a lower level.

Only parts of the user contour are going to be exposed to the explore engine in accordance with a user's own privacy settings. A explore engine wrapper is developed on the server aspect to include a partial user contour with the results obtained from a explore engine. Rankings from each partial user contours and explore engine results square measure combined. The bespoke results square measure delivered to the user by the wrapper.

The solution has 3 parts: initial, a climbable algorithmic program mechanically builds a class-conscious user contour from offered supply knowledge. Then, privacy parameters square measure offered to the user to work out the content and quantity of private knowledge that may be unconcealed. Third, a explore engine wrapper personalizes the explore results with the assistance of the partial user contour

Blue Eyes Intelligence Engineering 


\section{PRIVACY-ENHANCING PERSONIFICATIONEXPLORE}

Constructing a class-conscious User Contour Any personal documents like browsing history and emails on a user's pc may be the info supply for user contours. Our hypothesis is that terms that regularly seem in such documents represent topics that interest users. This concentrate on frequent terms limits the spatiality of the document set, that any provides a transparent description of users' interest.

This approach proposes to make a class-conscious user contour supported frequent terms. within the hierarchy, general terms with higher frequency square measure placed at higher levels, and specific terms with lower frequency square measure placed at lower levels. 4.2 measure Privacy in keeping with Alan Westin, "privacy is that the claim of people, groups, or establishments to work out for themselves once, however and to what extent knowledge is communicated to others". Privacy intrinsically is regarding protective users' personal knowledge. However, it's users' management that includes the justification of privacy.

With the entire user contour made higher than, AN approach with none privacy risk is to grant users full management over the terms within the hierarchy in order that they'll favor to hide any terms manually as they need. sadly, studies have shown that the overwhelming majority of users square measure continuously reluctant to produce any express input on their interests.

Interestingly, this live matches dead with our following observations on users' privacy concern: the interest with giant self-data corresponds to 2 styles of knowledge to that users square measure sometimes sensitive to grant access to. the primary case is that the interest itself is simply too specific. Users may not mind telling others regarding general interests, i.e. a user likes basketball, however is cautious regarding rental others apprehend his weekly basketball schedule.

The second case is that the interest is general however less fashionable among all interests. it would represent a personal event. the thought is to safeguard non-public knowledge that's either too specific or too sensitive within the user contour. each types may be measured by the support of the interest, below the belief that the additional specific or sensitive the interest is that the larger self-data the interest can carry. This ends up in the 2 parameters for specifying the need of privacy protection.

\section{USERSCUSTOMIZABLE PRIVACYPRESERVINGEXPLORE (UPS)}

The Personification net Explore (PWS) theme is increased to manage topic relationship based mostly knowledgeable attacks. The User customizable Privacy protective Explore (UPS) model is increased to resist question session based mostly attacks. question generalization is performed with question priority values. Anonymisation and topic taxonomy models square measure wont to improve the personification method.

The system is meant to safeguard the online personification theme with attack dominant mechanism. Privacy is ensured with Anonymisation strategies. question optimisation method is use to boost the question values. The system is split into six major modules. they're question log instrument, user contour construction and question generalization mistreatment GreedyDP, question generalization mistreatment GreedyIL, personification explore method and attack controller. The question log instrument module is meant to perform preprocess on user question logs. User question contours square measure made mistreatment question keywords.

Query values square measure generalized below the question generalization with GreedyDP module. question values square measure generalized below the question generalization with GreedyIL module. question optimisation method is disbursed below the personification explore method module. question session attacks square measure handled in attack controller module. I question Log instrument User question values square measure maintained below the question log files. User and question details square measure parsed from the question log knowledge.

Redundant $\log$ entries square measure off from the $\log$ knowledge. Optimized question knowledge values square measure updated into the information. II User Contour Construction User contours square measure made to manage the explore behavior of the user. Explore history is employed within the user contour construction method. question keywords square measure updated with the frequency values. Domain knowledge is updated with the explore question values.

III question Generalization mistreatment GreedyDP Anonymisation strategies square measure wont to offer privacy for user question values. User question values square measure generalized for privacy preservation. Greedy discriminating power (GreedyDP) algorithmic program is employed for the question generalization method. Generalized question values square measure updated within the user explore history setting. IV question Generalization mistreatment GreedyIL question values square measure generalized with knowledge lose factors.

Greedy knowledge Loss (GreedyIL) algorithmic program is employed for the generalization method. knowledge usage is taken into account within the generalization method. Generalized question keywords square measure utilized in the explore optimisation method. V Personification Explore method Privacy preserved net explore is performed within the personification explore method.

Query optimisation is employed to boost the question keywords. Generalized question keywords square measure utilized in the question optimisation method. question weight values square measure used for the question optimisation method. VI Attack Controller The attack controller is employed to manage question attacks. Session knowledge square measure protected to manage session based mostly attacks.

\section{CONCLUSION}

Additionally, UPS additionally performed on-line generalization on user contours to safeguard the non-public privacy while not compromising the explore quality. wishing on the definition of 2 conflicting metrics, specifically personification utility and privacy risk, for class-conscious user contour, we tend to 
formulate the matter of privacy-preserving personification explore as ??-Risk Contour Generalization, with its NP-hardness verified.

We projected 2 easy however effective generalization algorithms, GreedyDP and GreedyIL, to support runtime identification. whereas the previous tries to maximise the discriminating power (DP), the latter tries to attenuate the info loss (IL). Personification net explore (PWS) is employed to boost the standard of assorted explore services on the net. Privacy preserved PWS strategies square knowledge in explore method. User ustomizable Privacy-

\section{REFERENCES}

1. J.S. Breese, D. Heckerman, and C.M. Kadie, "Empirical Analysis ofPredictive Algorithms for Collaborative Filtering," Proc. 14th Conf.Uncertainty in Artificial Intelligence (UAI), pp. 43-52, 1998.

2. P.A. Chirita, W. Nejdl, R. Paiu, and C. Kohlschu" tter, "Using ODPMetadata to Personalize Explore," Proc. 28th Ann. Int'l ACM SIGIRConf. Reexplore and Development Information Retrieval (SIGIR), 2005.

3. A. Pretschner and S. Gauch, "Ontology-Based Personalized Exploreand Browsing," Proc. IEEE 11 th Int'1 Conf. Tools with ArtificialIntelligence (ICTAI '99), 1999 